\title{
INTERACTION OF FLUX TUBES WITH SOUND WAVES
}

\author{
C UBEROI \\ Department of Applied Mathematics \\ Indian Institute of Science \\ Bangalore 560012 \\ INDIA
}

\begin{abstract}
The Landau damping of sound waves in a plasma consisting of ensemble of magnetic flux tubes is discussed. It is shown that sound waves cannot be Landau damped in general but under certain restricted conditions on plasma parameters the possibility of absorption of these waves can exist. The possibility of radiative damping of the acoustic waves along the magnetic filaments is also discussed. It appears that the most plausible mechanism of damping of sound waves in a plasma consisting of magnetic filaments can be due to scattering of a sound wave by the filaments.
\end{abstract}

In a recent paper [1], from the point of view of looking at the possibility of confining hot plasma by a cold gas blanket Uberoi and Somasundaram [1] discussed the dispersion characteristics of an Alfvén surface wave along a cylindrical plasma column embedded in a neutral gas by discussing the phase velocity of the surface waves as a function of the parameter $\alpha=v_{A} / s$, where $v_{A}$ is the Alfvén speed in the plasma column and $s$ is the sound speed of the surrounding neutral medium. In this paper I would like to reinterpret the results obtained from this parametric study of the dispersion equation to discuss some aspects of sound wave interaction with flux tubes in the solar plasma. This work is an extension of the preliminary study given in an earlier paper [2].

Consider a cylindrical plasma column of radius ' $a$ ' with mass density $\rho_{0}$ and an internal magnetic field $\mathbf{B}_{0}$ surrounded by a compressible gas of mass density $\rho_{\mathrm{g} 0}$ and magnetic field $\mathbf{B}_{\mathrm{g} 0 \text {. Both }} \mathbf{B}_{0}$ and $\mathbf{B}_{\mathrm{g} 0}$ are directed along the axis of the column. The dispersion relation obtained by solving the linearized MHD equations inside the flux tube, the equations for the outer neutral gas and matching the solutions with the help of boundary conditions at $r=a$ is given as:

$$
\frac{v_{\mathrm{ph}}^{2}}{v_{A}^{2}}\left[1+\eta \frac{h_{m}(k a) g_{m}(\tau a)}{\left(1-\alpha^{2} v_{p h}^{2} / v_{A}^{2}\right)}\right]-\beta^{2} F_{m}(k a)-1=0,
$$

where

$$
\begin{aligned}
& h_{m}(k a)=I_{m}^{\prime}(k a) / I_{m}(k a) \\
& g_{m}(\tau a)=-K_{m}(\tau a) / K_{m}^{\prime}(\tau a) \\
& F_{m}(k a)=-I_{m}^{\prime}(k a) K_{m}(k a) / K_{m}^{\prime}(k a) I_{m}(k a) .
\end{aligned}
$$

E. R. Priest and V. Krishan (eds.), Basic Plasma Processes on the Sun, 239-243.

(C) 1990 IAU. Printed in the Netherlands. 
$\mathrm{v}_{\mathrm{ph}}=\omega / \mathrm{k}, \alpha=\mathrm{v}_{\mathrm{A}} / \mathrm{s}, \eta=\rho_{\mathrm{g} 0} / \rho_{0}, \beta=\mathrm{B}_{\mathrm{g} 0} / \mathrm{B}_{0}$ are the interface parameters and $\tau \alpha=\kappa \alpha(1-$ $\left.v_{\mathrm{ph}}^{2} / \mathrm{s}^{2}\right)^{1 / 2}$.

Without going into details, which are discussed in [1], I like to point out the following important results obtained in the parametric study of dispersion equation (1). The dispersion characteristics for $\alpha<1$ do not show any change from those obtained for the plasma-plasma interface [3]. But, when $\alpha>1$ many interesting features of the surface wave propagation are obtained. It is seen that $v_{p h} \sim s$ only for values of $\alpha>1$. So, the acoustic surface waves are generated along the plasma column surrounded by a compressible gas only when the strength of the magnetic field is such that $v_{A}>s$. As $\eta$ is increased, i.e. $\rho_{\mathrm{g} 0}$ increases or the neutral gas pressure increases, the value of $\alpha$ for which $\mathrm{v}_{\mathrm{ph}} \sim \mathrm{s}$ increases. Thus, the critical magnetic field $B_{0}$, above which Alfvén surface waves do not exist but acoustic surface waves can be generated increases with increasing gas pressure. In a subsequent paper [4] it was found that for large ka, when curvature effects are negligible, the critical magnetic field for low $\eta$ say $\eta=0.1$ or $\rho_{g} 0=0.1 \rho_{0}$ above which Alfvén surface waves do not exist is given by $\mathrm{v}_{\mathrm{A}} \sim \mathrm{s}$ and for $\rho_{\mathrm{g} 0}=0.5 \rho_{0}$ it is given by $\mathrm{v}_{\mathrm{A}}$ $\sim 1$.6s. For example if $\rho_{0}=10^{-7} \mathrm{gm} / \mathrm{cm}^{3}$ and $\mathrm{s}=15 \mathrm{~km} / \mathrm{s}$ in the neutral gas it can be shown that for $\eta=0.1$ which gives $\rho_{g 0}=0.1 \rho_{0}$ and gas pressure $P_{g 0}=0.105 \times 10^{2}$ Torr, the critical value of the magnetic field above which sound waves are excited is given as $B \sim$ 1680G when $\eta=0.5, \mathrm{P}_{\mathrm{g} 0}=0.53 \times 10^{2}$ Torr, $\mathrm{B}=2690 \mathrm{G}$. Similarly for $\eta=0.8, \mathrm{P}_{\mathrm{g} 0}=$ $0.84 \times 10^{2}$ Torr, $\mathrm{B} \sim 3030 \mathrm{G}$. Thus, for flux tubes with magnetic fields lying say in the range of 1000-2000 Gauss, for a given value of $\eta=0.1$ the flux tubes which can support sound surface waves will be in the narrow range 1680-2000G. For higher values of $\eta \geq$ 0.5 , no resonant tube will exist. I would like to point out here that in a preliminary work where the compressibility of the medium inside the flux tube is taken into account it is found that the value of $\alpha$ for which $\mathrm{v}_{\mathrm{ph}} \sim \mathrm{s}$ increases when $\mathrm{v}_{\mathrm{A}} / \mathrm{c}$ increases or $\mathrm{s}$ decreases.

The effect of curvature makes the Alfvén surface waves dispersive [3]. In order to understand the effect of dispersion due to curvature on the transformation of Alfvén to surface sound waves Uberoi and Somasundaram [1] study the dispersion characteristics of Alfvén surface waves for $m=0$ and $m= \pm 1$ modes as function of $\alpha$ for different values of the wave number ka. They find that due to the curvature effects for magnetic fields above the critical magnetic field such that $\alpha>1$, there exists a lower cut-off wave number $k_{c}=k_{c}$ $(\alpha, \eta, m, \beta)$, such that Alfvén surface waves do not exist for $k<k_{c}$. Hence, the surface sound waves for a cylindrical plasma will be restricted to the wavelength region $0<\lambda<\lambda_{c}$.

It was also seen that the value of $k_{c}$ increases when the magnetic field above the critical value, is increased but, decreases with increasing $\eta$. Therefore, for large gas pressures $\mathbf{k}_{\boldsymbol{c}}$ can be made small, i.e. sound waves will be of long wavelengths. For the axisymmetric modes, the value of $k_{c}$ is large compared to that of $m=0$ modes for any given value of the interface parameters.

In the solar or stellar convection zone and chromospheric atmosphere one meets with the situation when an ensemble of magnetic flux tubes concentrated in the plasma are embedded in the neutral medium lying far from one another. Sound wave propagation in the system consisting of 'magnetic filaments' was first considered by Ryutov and Ryutova [5] and more recently by Bogdan and Zweibel [6] and Bogdan [7]. Ryutov and Ryutova 
[5] by drawing the analogy of such a system to a liquid with gas bubbles, study on the basis of the magnetohydrodynamic equations the long wavelength sound oscillations of such a system. They note a very interesting property of this system: for long wave lengths $\lambda \gg i$, where $i$ is the average distance between the tubes, even if there are no dissipative processes, the sound oscillations are absorbed because of an effect that is in a certain sense analogous to Landau damping in a rarefied plasma and which consists of the resonant excitation of flexure waves that propagate along the magnetic filaments. The Landau damping of sound oscillations arises due to resonance interaction of sound oscillations, of the flux tubes. They find that the Landau damping rate $v_{i} \sim \mathbf{k f s}$, where $f$ is the total fraction of the volume occupied by flux tubes. As they consider the tubes with radius a lying far from one another $f \approx a^{2} / i^{2}<<1$. They also calculate the radiation damping of the surface oscillations of the flux tubes and find the damping rate $v_{\text {rad }} \sim k^{2} a^{2}$. The damping rate due to scattering is also calculated as $v_{\text {scatt }} \sim \mathrm{ks}(\mathrm{ka})^{4} / \mathrm{f}$. Comparing the damping rates $v_{i}, v_{\mathrm{rad}}$ and $v_{\text {scatt }}$ it is easily seen that for long wavelengths $\lambda \gg \mathrm{i}, v_{i}>v_{\text {rad }}$ and $v_{\text {scatt }}$ so the important source by which sound wave energy can be absorbed by the plasma system is the Landau damping of the sound waves arising due to resonant excitation of surface waves along the flux tubes. The energy of the sound waves thus gets transferred to the energy of the filament oscillations over a period of time of order $v_{i}^{-1}$; over a considerably longer time

of the order of $1 / \mathrm{v}_{\text {rad }}$ the filaments give off their energy through secondary sound waves.

Using the results obtained for the surface wave propagation along a single magnetic flux tube as reported above we show that the possibility of Landau damping of the acoustic waves can exist only under restricted conditions on plasma parameters. For a cylindrical plasma column surrounded by a neutral gas it is seen that acoustic waves along the magnetic filaments will exist when magnetic fields are above the critical magnetic field only for wavelengths $\lambda<\lambda_{c}$. Therefore only if $\lambda_{c} \gg \mathrm{i}$, is the Landau damping of acoustic waves possible. The value of $\lambda_{c}$ depends on various plasma parameters. As shown by Uberoi and Somasundaram [1], the value of $k_{c}$ can be made very small, i.e. sound waves will be of long wavelengths, for large values of the neutral gas pressure only but then the value of the critical magnetic field above which flux tubes will be of resonating class also increases with higher values of the gas pressure thus restricting the general generation of the sound waves. The possibility of Landau damping and absorption of sound wave energy by this mechanism thus is very much restricted and cannot exist in general as discussed by Ryutov and Ryutova (1976).

In the opposite limit, when $\mathrm{ka} \ll 1<k \mathrm{ki}$, both $v_{\mathrm{i}}$ and $v_{\mathrm{rad}}$ are very small, so damping of the sound waves is mainly determined by the scattering. The resonant scattering again will depend on the critical magnetic field. The energy transfer of acoustic waves to a plasma embedded with flux tubes thus depends very critically on the strength of the magnetic field of the flux tubes and the values of the interface parameters.

\section{References}

1. Uberoi, C and Somasundaram, K (1982), 'Alfvén Surface Waves along a cylindrical plasma column surrounded by a neutral gas', Plasma Physics, 24, 465-474.

2. Uberoi, C (1984) 'On the Landau damping of sound waves in a plasma physics', 26, 1037. 
3. Uberoi, C and Somasundaram, K (1982), 'Acoustic surface wave generation along a cylindrical plasma column surrounded by a compressible gas', Phys. Rev. A., 25, 2414-2416.

4. Uberoi, C and Somasundaram, K (1980), 'Alfvén Surface Waves along cylindrical plasma columns', Plasma Physics, 22, 747-752.

5. Ryutov, D D and Ryutova, M P (1976) 'Sound oscillations in a plasma with "magnetic filaments"', Sov. Phys. JETP. 43, 491-497.

6. Bogdan, T J and Zweibel, E G (1987), 'Propagation of compressible waves through fibril magnetic fields', $A p$. J., 312, 444-456.

7. Bogdan, T J (1989) 'On the resonance scattering of sound by slender magnetic flux tubes', Ap. J. 345, 1042-1049.

\section{DISCUSSION}

FORBES: I thought you were going to propose that resonant absorption would affect reconnection by heating the plasma and so reducing the electrical conductivity. However, you seem to be proposing that the concentration of surface waves in a singular layer will drive the tearing-mode.

UBEROI: Yes, the final picture of my analysis is that the surface waves can drive magnetic reconnection across the neutral layer by inducing the tearing mode instability. But this kind of reconnection is associated with the Alfvén resonance process. The higher frequencies of the excited spectrum of surface waves can give rise to resonant coupling, whereas the low frequencies produce reconnection.

PRIEST: How does your analysis differ from that of Sakai et al (Solar Phys 91,103,1984), who showed (in connection with solar flares) that tearing mode instability could be driven faster than normal by waves incident on a current sheet?

UBEROI: I have not seen Sakai's paper. In my work I model surface-wave-driven magnetic reconnection by using the analysis of Alfvén waves propagating in a system with resonant layers.

HOLLWEG: (i) In my description of resonance absorption, I consider driven harmonic oscillator equations. Are your "well-behaved" solutions just the homogeneous solutions to my oscillator equations?

(ii) Is your temporal increase of z-current density simply due to phase-mixing?

UBEROI: (i) I appreciate your physical description of resonance absorption, though I am not sure the harmonic oscillator equation is equivalent to the Alfvén wave equation.

(ii) The temporal increase of z-current density is not due to phase-mixing. This increase is for time intervals $0<\mathrm{t}<\mathrm{t}^{*}$, and it is only for $\mathrm{t}>\mathrm{t}^{*}$ that phase-mixing begins.

ROBERTS: Why did you say that your solution holds only for a finite amount of time $t$ ? That limitation was not clear to me. I would have expected your solution to hold for all time, even if some quantities grow secularly, provided one took a sufficiently small ampitude for linear theory to be valid. 
UBEROI: The well-behaved solutions only hold good in an interval $0<\mathrm{t}<\mathrm{t}^{*}$, where $\mathrm{t}^{*}$ is calculated from the phase-mixing time. Even if the solutions are of small amplitude and obtained by linear theory, the effect of the continuous spectrum is to give rise to phase mixing for $t>t^{*}$. Once the phase mixing starts there is no meaningful well-behaved solution for the Alfvén wave equation.

RYUTOVA: The paper [5] mentioned by C. Uberoi was apparently the first to consider sound wave propagation in a plasma containing an ensemble of randomly distributed magnetic flux tubes. The problem of flexure oscillations of a single flux tube is also considered there, including radiative damping and resonance scattering. Uberoi's paper represents an important step in generalising this work. We found in agreement with $\mathrm{C}$. Uberoi that for short wavelength acoustic waves their damping is determined by the resonant scattering. Moreover, the nonlinear estimates were made for the maximum energy which can be transferred by a magnetic flux tube due to the interaction (resonance absorption or scattering) with a finite-amplitude sound wave. 